\title{
Thermoelectroluminescence in thin films of zinc and copper phthalocyanines
}

\author{
L N TRIPATHI and Y MISRA \\ Department of Physics, University of Gorakhpur, Gorakhpur 273001, India
}

\begin{abstract}
Thermoelectroluminescence studies were performed on zinc phthalocyanine and copper phthalocyanine films deposited on transparent and conducting glass substrate. The EL glow curves were recorded. The trap depths and frequency factors have also been determined using three different methods.
\end{abstract}

Keywords. Thermoelectroluminescence; zinc phthalocyanines; copper phthalocyanines

\section{Introduction}

Thermoelectroluminescence ( $\mathrm{TEL}$ ) is one of the thermally stimulated processes to study the thermal activation of inorganic and organic phosphors under a super-imposed d.c. or a.c. electric fields. This method gives information about the traps and their distribution like the simple glow curve experiment, viz thermoluminescence (TL) or thermally stimulated current ( TSC) or thermally stimulated electron emission (TSEE) experiments. Trapping action in EL processes can be understood by studying the EL brightness as a function of temperature.

In organic hydrocarbons, the temperature dependence of space charge limited current in d.c. electroluminescence has been studied in anthracene (Nakada and Ishahara 1964; Bierman 1963) and metal-free phthalocyanines (Heilmeir and Warfeld 1963). Thermally stimulated current measurements on the trapping levels of copper phthalocyanine have been reported by Hoshino (1981). No attempt, has, however, been made to determine parameters such as trap depths and recombination rate constants or frequency factors in $\mathrm{ZnPc}$ and $\mathrm{CuPc}$. The present study attempts to record the TEL glow peaks for these materials to determine the above parameters.

\section{Theoretical}

EL brightness is strongly dependent on the variation in temperature, which in turn brings about a number of changes; for example, electrons/holes are detrapped or released and the mean trapping time of the carriers is also affected. The degree of ionization of the trap levels also affects the potential in the space charge region. The increase of brightness at a particular field frequency may be due to release of electrons/holes at a particular temperature, which interact with excitons leading to enhanced EL. These electrons may associate themselves with holes supplied from the positive contact forming excitons which decay radiatively giving EL emission. These carriers released from the traps are over and above those which are injected from the 
electrode contacts during the positive half cycles and which eventually form spacecharge region leading to the formation of excitons. The release from traps, therefore, depends both on temperature and time. It therefore becomes necessary to consider the effect of temperature and the frequency of the alternating voltage together.

Chukova (1972), Johnson et al (1956) and Haake (1955) showed that the effect of temperature and frequency on eL brightness can be expressed by the relation

$$
f=S \exp (-E / k T),
$$

where $E$ is the trap activation energy, $\mathrm{S}$ the frequency factor or carrier attempt to escape frequency, $f$ the frequency of the electric field and $T$, the temperature at which the brightness is maximum.

If the experiment is performed at a number of frequencies $f_{1}, f_{2}, f_{3} \ldots$ at a constant fixed voltage and $T_{1}, T_{2}, T_{3} \ldots$ be the corresponding temperatures of maximum brightness for a particular peak, then the slope of the line drawn between $\log f$ and $1 / T$ would give $-E / K$ and the intercept on the $\log f$ axis would give logs. From these data, $E$ and $S$ could be found out.

There is a second approach to determine the trap depth. If the experiment is performed at different heating rates, a particular peak is shifted towards the higher temperature side at a higher rate of heating. The shift in EL brightness is also expected with change in frequency of the field. At higher frequencies the peak would shift towards higher temperature side. Therefore, the different maxima at a particular frequency $f$, can be represented by an equation similar to (1)

$$
f_{1}=S_{n} \exp \left(-E_{n} / k T_{n}\right),
$$

where $E_{n}$ is the energy of the $n$th trap corresponding to temperature $T_{n}$ and $S_{n}$ is the frequency factor for that trap.

If the experiment is repeated at another frequency $f_{2}$ but at the same voltage, then

$$
f_{2}=S_{n} \exp \left(-E_{n} / k T_{n}^{\prime}\right),
$$

where $T_{n}^{\prime}$ is the new temperature at which the new peak maxima occur. From (2) and (3), we have

$$
E_{n}=K \frac{\left(\log f_{1}-\log f_{2}\right)}{\left(\frac{1}{T_{n}^{\prime}}-\frac{1}{T_{n}}\right)}
$$

$S$ can now be calculated from (2) and (3).

We use a third method known as the initial rise method to determine trap depth. Here, the probability that the electrons/holes will be excited by thermal energy is proportional to $\exp (-E / k T)$. At lower temperatures, a smaller number of electrons/holes will be released and their number will increase with increase in temperature. After reaching a maximum, it falls as the traps are depleted. The resulting TL curve has a single peak, the peak maxima denoting the depth of the level. If $\log I$ (at a constant frequency and voltage) versus $1 / T$ is plotted, we could get a straight line, which would give the trap depth. The peak shape and the temperature of peak maxima depend not only on the intrinsic parameters such as $E$ and $S$, but also on the heating rate, the intensity of EL emission and also on the concentration of traps and excitons. Several types of traps with different activation energies usually result in several $\mathrm{TL}$ peaks which 
may superimpose. There may be as many glow peaks as the number of trap levels in the TEL curve. The brightness maxima correspond to the exhaustion of certain group of trap levels.

\section{Measuring arrangement and procedure}

All the TEL curves were recorded above $200^{\circ} \mathrm{K}$. The phosphor samples were in the form of thin films. The EL cell was fixed on a metallic plate of the cryostat, over which a heating element was wound, thin mica sheet providing an insulation.

The temperature of the EL cell was measured by a chromel-alumel thermocouple. One junction of the thermocouple was securely fixed in the metallic electrode of the cell using thin mica insulation and the other junction was dipped in ice. The EMF generated in the thermocouple was recorded by a digital multimeter and the value of corresponding temperature was computed from the standard table with an accuracy of $1^{\circ} \mathrm{C}$. The heating rate was adjusted at $0.68^{\circ} \mathrm{K} / \mathrm{sec}$ by controlling the current in the heating coil.

To obtain EL, the cell terminal was connected to a wide band amplifier capable of giving a.c. voltages upto $1000 \mathrm{~V}$ and frequencies from $1 \mathrm{~Hz}$ to $10 \mathrm{kHz}$. The integrated light output was measured using photomultiplier tube (1P21) operated by a stabilized power supply unit giving voltages upto $1200 \mathrm{~V}$. The output terminals of the photomultiplier tube were connected to a multiflex galvanometer of current sensitivity $10^{-9} \mathrm{amp} / \mathrm{mm}$ to record the relative intensity in arbitrary scale.

For recording TEL emission, $500 \mathrm{~V}$ (r.m.s.) was applied between the plate of the EL cell and the heating was then started. The light output was recorded at 3 field frequencies, $1.5,2.0$ and $2.5 \mathrm{kHz}$ respectively for every sample.

\section{Results and discussion}

The TEL glow curves for $\mathrm{ZnPc}$ and $\mathrm{CuPc}$ are shown in figures 1 and 2 respectively. The maxima of the brightness temperature curves are seen to shift to lower temperatures with increasing field frequencies. In inorganic systems so far known, the peak maxima are reported to shift towards the higher temperature side (Tripathi et al 1982). Normally such systems have a majority of electron traps. This probably implies that in $\mathrm{ZnPc}$ and $\mathrm{CuPc}$, the presence of hole traps might cause this behaviour.

The $\log f$ versus $1 / T$ plots for each sample show a straight line. This gives the trap depth directly and intercept the value of $S$. The calculated values of $E$ and $S$ are shown in table 1 for each system.

Trap depths have also been calculated using equation (4) at three different frequencies $1.5,2.0$ and $2.5 \mathrm{kHz}$ and the results are presented in table 1 . The initial rise method $(\log B$ versus $1 / T)$ curves are shown in figures 5 and 6 and the values of $E$ are shown in table 1. The value of $S$ for each sample is of the order of $10^{6} \mathrm{sec}^{-1}$.

Hwang and Kao (1974) studied the d.c. electroluminescence and its temperature dependence in anthracene single crystals and reported one glow peak maxima at $313^{\circ} \mathrm{K}$. This has been interpreted as arising due to competing behaviour of two types of processes: (i) due to exciton-trapped-exciton interaction and (ii) due to exciton carrier interaction. The exciton-trapped-exciton interaction dominates at temperatures lower 


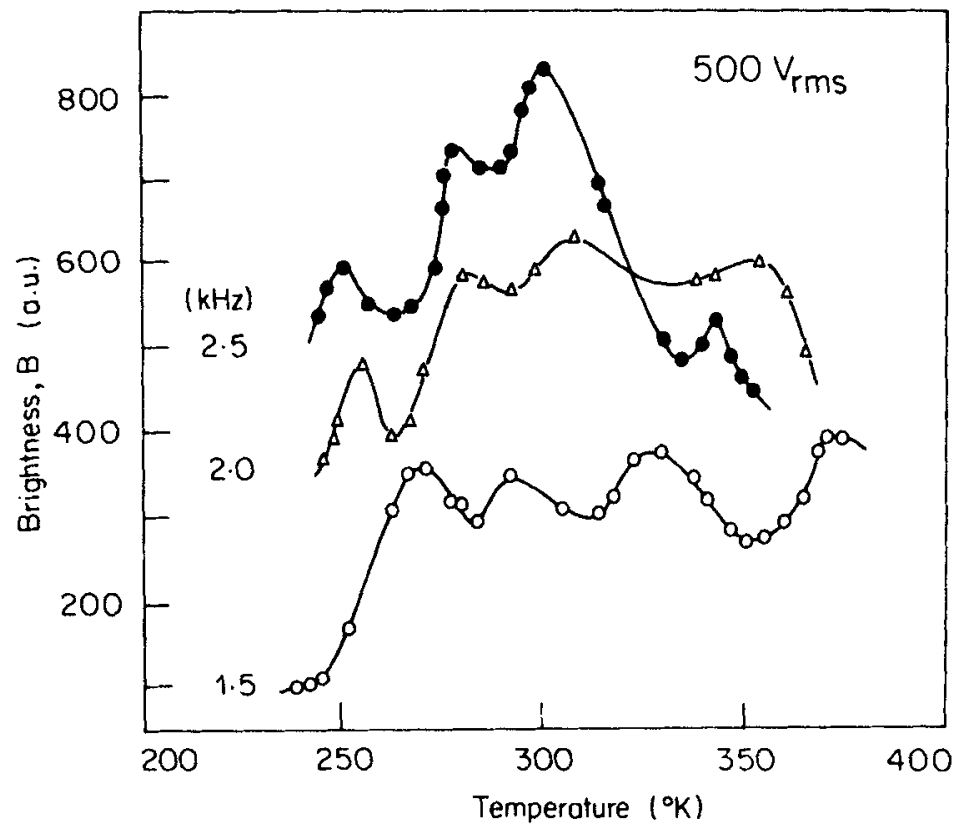

Figure 1. TEL curves for $\mathrm{ZnPc}(150 \mathrm{~nm}$ thick).

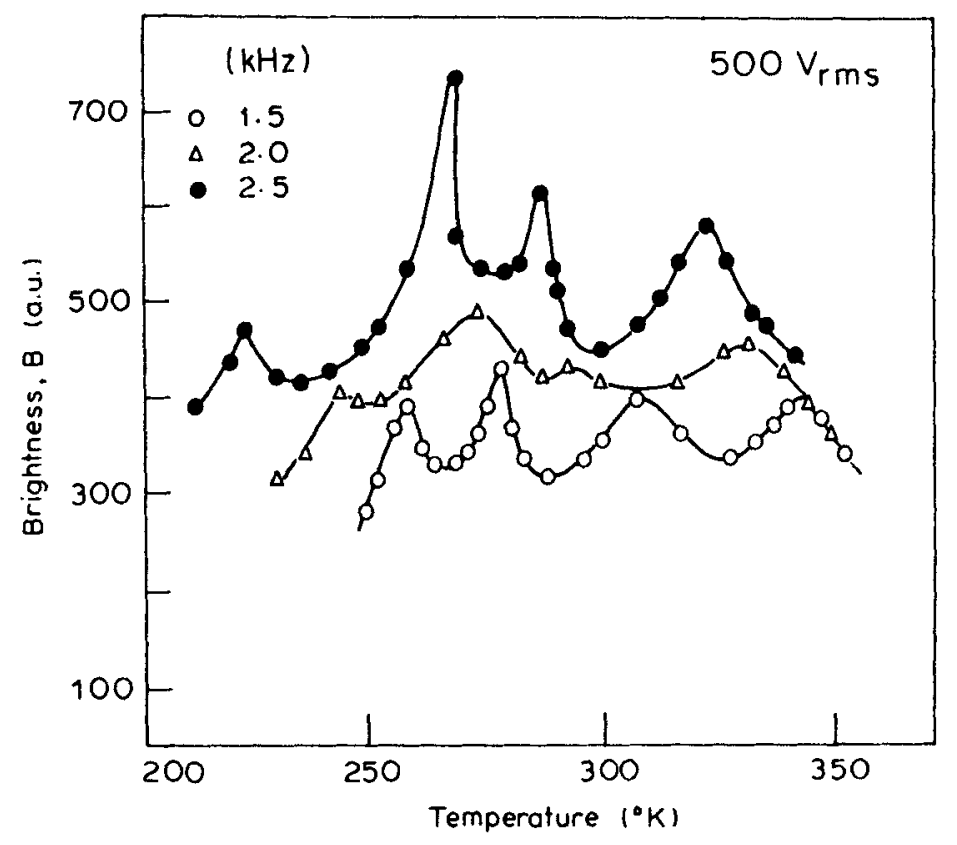

Figure 2. TEL curves for CuPc film (200 nm thick). 


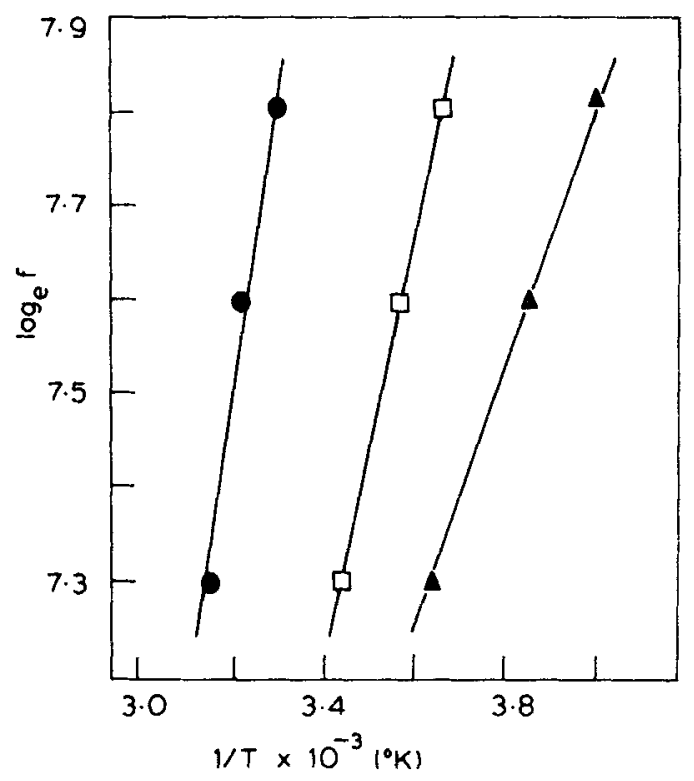

Figure 3. $\log f$ vs $1 / T$ for $\mathrm{ZnPc}$ film (200 nm thick).

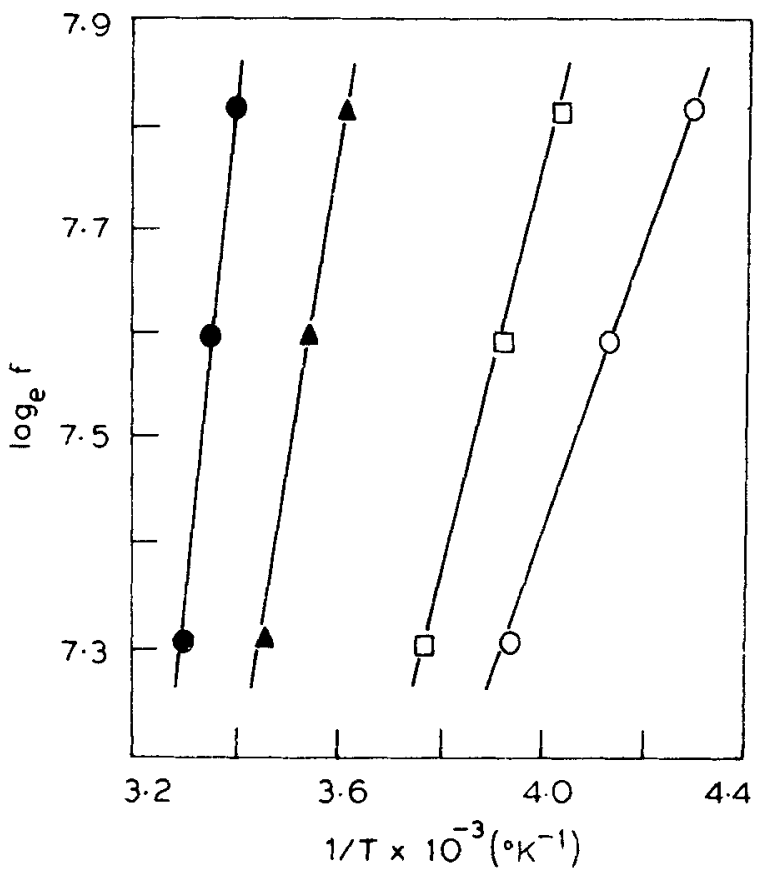

Figure 4. $\log f$ vs $1 / T$ for CuPc film (200 nm thick) 
Table 1. Values of trap depths $E$ and the frequency factor $S \mathrm{ZnPc}$ and $\mathrm{CuPc}$

\begin{tabular}{|c|c|c|c|c|c|c|}
\hline \multirow{2}{*}{$\begin{array}{l}\text { Phosphor } \\
\text { film }\end{array}$} & \multicolumn{3}{|c|}{$\begin{array}{l}\text { From glow curve } \\
\text { at low temperature } \\
\text { trap depth }(\mathrm{eV})\end{array}$} & \multirow{2}{*}{$\begin{array}{l}\text { Trap depth } \\
\quad(\text { TEL) } \\
\log f \text { vs } 1 / T\end{array}$} & \multirow{2}{*}{$\begin{array}{c}\text { Trap depth } \\
\log B \text { vs } 1 / T\end{array}$} & \multirow{2}{*}{$\begin{array}{c}\text { Escape } \\
\text { frequency } \\
\left(\sec ^{-1}\right)\end{array}$} \\
\hline & $f_{1}-f_{2}$ & $f_{2}-f_{3}$ & $f_{3}-f_{4}$ & & & \\
\hline \multirow[t]{3}{*}{$\mathrm{ZnPc}$} & $0 \cdot 10$ & 0.12 & 0.11 & $0 \cdot 14$ & $0 \cdot 16$ & \multirow{3}{*}{$10^{6}$} \\
\hline & 0.21 & 0.21 & 0.21 & 0.22 & 0.23 & \\
\hline & 0.50 & 0.47 & 0.49 & 0.26 & 0.27 & \\
\hline \multirow[t]{4}{*}{$\mathrm{CuPc}$} & 0.14 & 0.07 & 0.08 & 0.13 & $0 \cdot 16$ & \multirow{4}{*}{$10^{6}$} \\
\hline & $0 \cdot 16$ & 0.19 & 0.17 & 0.16 & $0 \cdot 18$ & \\
\hline & 0.36 & 0.27 & 0.29 & 0.24 & 0.25 & \\
\hline & 0.83 & $1 \cdot 16$ & - & 0.74 & - & \\
\hline
\end{tabular}

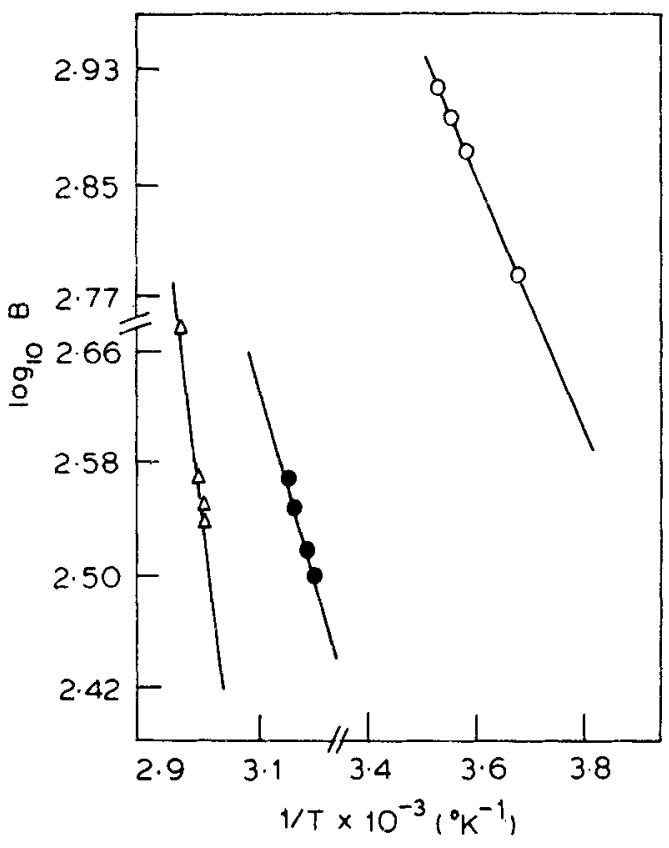

Figure 5. $\log B$ vs $1 / T$ for $\mathrm{ZnPc}$.

than the maximum while the exciton carrier interaction dominates at higher temperatures.

In the temperature range shown, we find four peaks corresponding to 4 groups of traps in both the materials.

It may be pointed out that in EL experiment, the phosphors are excited by photons at low temperatures for some time to fill the trap levels with electrons/holes and later the temperature is raised to get the stored energy released. But no such excitation was done in the present study. The EL radiation itself acts as excitation source for filling the traps 


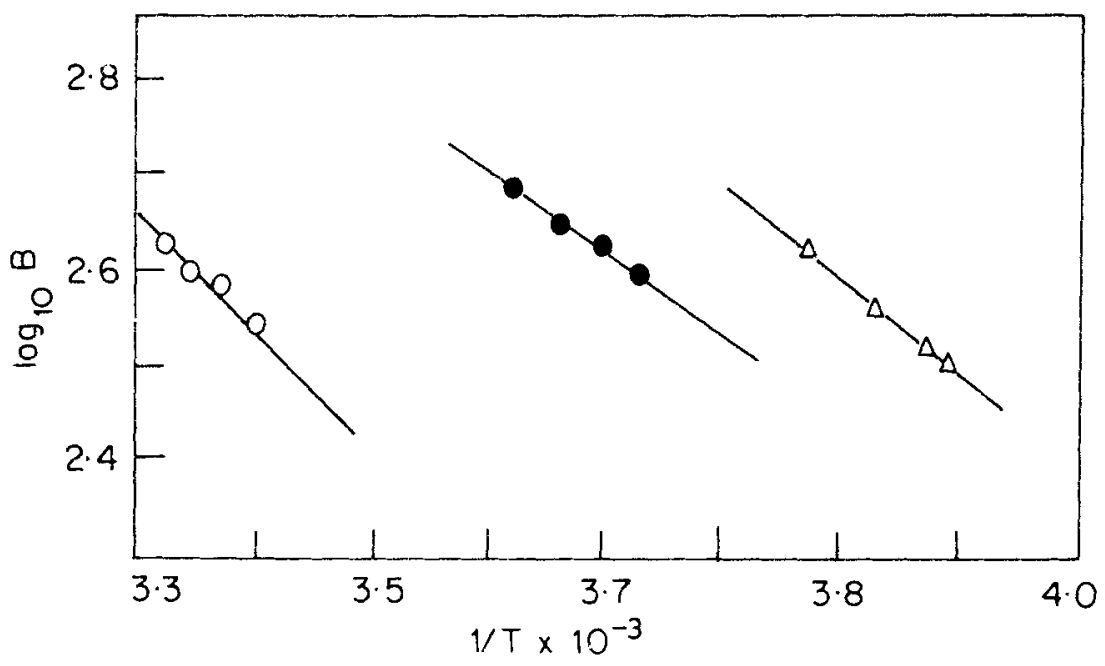

Figure 6. $\log \mathrm{B}$ vs $1 / T$ for $\mathrm{CuPc}$.

and these trapped electrons/holes are released by the combined action of field frequency and temperature.

\section{References}

Bierman A 1963 Phys. Rev. 1302226

Chukova Y P 1972 Electrolum. Proc., P. N. Lebedev Phys. Institute, 5097

Haake C H 1957 J. Electrochem. Soc. 104291

Heilmeir G H and Warfeld G 1963 J. Chem. Phys. 38163

Hoshino Y 1981 J. Appl. Phys. 525655

Hwang W and Kao K C 1974 J. Chem. Phys. 603845

Johnson P D, Piper W W and Williams F E 1956 J. Electrochem. Soc. 103221

Nakada I and Ishahara X 1964 J. Phys. Soc. Jpn 19695

Tripathi L N, Chaube B R and Misra C P 1982 Pramana (J. Phys.) 19385 